\title{
Analysis and Optimization of the Transmission Angle of Crank Rocker Mechanism
}

\author{
Sun Jian \\ Jiangsu Key Laboratory of Large Engineering Equipment Detection and Control, School of Mechanical \& Electrical \\ Engineering \\ Xuzhou Institute of Technology, \\ Xuzhou, China \\ e-mail: sunjian751@163.com
}

\begin{abstract}
In view of the quick return characteristics of I and II type crank rocker mechanism, the inner relationship between the size of components is analyzed. The parametric model of mechanism is established based on ADAMS software, then, with the minimum transmission angle to take the maximum as the optimization goal, lists the objective function and constraint equations of I and II type crank rocker mechanism. With the established model, the optimization of the structure parameters is proposed to recover the maximum value of the minimum transmission angle. By the optimization, the minimum transmission angle of mechanism get a great improvement, this result can effectively improve transmission efficiency of the mechanism.
\end{abstract}

Keywords-optimization; transmission angle; crank rocker mechanism; ADAMS; simulation

\section{INTRODUCTION}

For planar four-bar mechanism, when the sum of shortest and longest rod is less than or equal to the sum of other two rods, the mechanism exists crank[1]. At this time, when the neighbors rod of the shortest rod is crank, the institution known as crank rocker mechanism. Because the crank rocker mechanism with large bearing capacity and the use of the quick return characteristics can improve the production efficiency and other advantages, it has been widely used in mechanical engineering[2]. In mechanism design, the requirements of follower mechanism must satisfy a certain motion law, which requires the necessary analysis of mechanism motion[3]. There are many ways to analyze the mechanism motion law, and common analysis method is a graphical method and analytical method. However, the graphic method design has characteristics of low precision and time consuming, and computing workload of analytical method is big, which must deal with the aid of computer programming. In this paper, the motion characteristics of crank rocker mechanism is analyzed, and the parameterization model of crank rocker mechanism is established by ADAMS software for data analysis and optimization of the transmission angle, and then get the minimum transmission angle of crank rocker mechanism has a maximum of transmission performance exact solutions.

\section{WORKING PRINCIPLE}

As shown in Fig .1, in the crank rocker organization of the OABC, the mechanism mainly composed by crank, connecting rod, rocker and frame[4]. Where O, A, B and C are each link point. L1, L2, L3 and L4 represent the length of crank, connecting rod, rocker and the frame, respectively. Rocker swing angle $\angle \mathrm{B}_{1} \mathrm{CB}_{2}=\Psi$, Pole position angle $\angle \mathrm{B}_{1} \mathrm{OB}_{2}=\theta$, thus the travel velocity ratio coefficient can be obtained $\mathrm{K}=\left(180^{\circ}+\theta\right) /\left(180^{\circ}-\theta\right)$. $\angle \mathrm{A}_{3} \mathrm{~B}_{3} \mathrm{C}$ and $\angle \mathrm{A}_{4} \mathrm{~B}_{4} \mathrm{C}$ are the minimum angle $\delta_{\text {min }}$ and maximum angle $\delta_{\max }$ between the connecting rod and rocker, respectively. Regardless of the friction influence, the transmission Angle $\gamma_{\min }$ of crank rocker mechanism is the smaller values of $\delta_{\min }$ or $180^{\circ}-\delta_{\min }\left(\right.$ when $\left.\delta_{\min }>90^{\circ}\right)$.

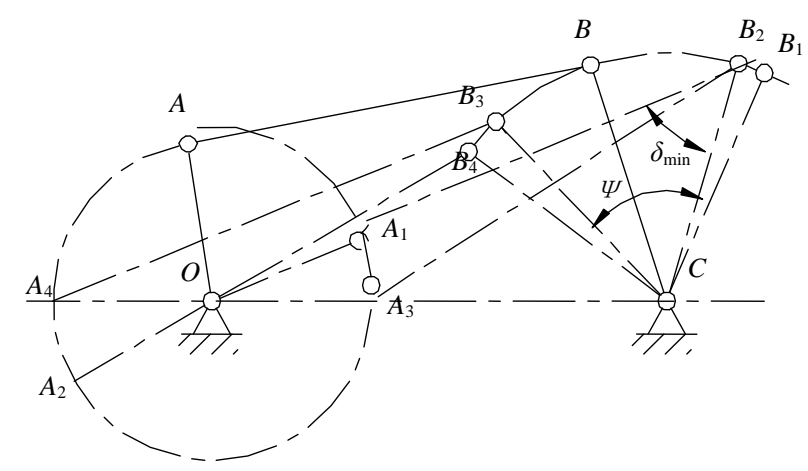

Figure 1. Schematic diagram of I type crank rocker mechanism

According to the motion characteristics, crank rocker mechanism can be divided into two types: I and II type mechanism[5]. Among them, the movement features of I type crank rocker mechanism is rocker slow stroke swinging direction same as the crank shift, and the structure characteristics is that $\mathrm{O}$ and $\mathrm{C}$ point are located on the same side of line $t-t$ through the $B_{1}$ and $B_{2}$, and the relationship between the component size is $L_{1}^{2}+L_{4}^{2}<L_{2}^{2}+L_{3}^{2}$, as shown in Fig .1. The movement features of II type crank rocker mechanism is rocker slow stroke swinging direction opposite with the crank shift, and the structure characteristics is that $\mathrm{O}$ and $\mathrm{C}$ point are located on the different side of line $\mathrm{t}-\mathrm{t}$ through the $\mathrm{B} 1$ and $\mathrm{B} 2$, and the relationship between the component size is $L_{1}^{2}+L_{4}^{2}>L_{2}^{2}+L_{3}^{2}$, as shown in Fig .2.

The relation differences of swing direction of rocker slow stroke with crank determine relations determine the 
differences applications of I and II type of crank rocker mechanism, and the differences of structure characteristics with size relationship also make the relative degree and movement characteristics of quick return characteristics differences have quite different[6].

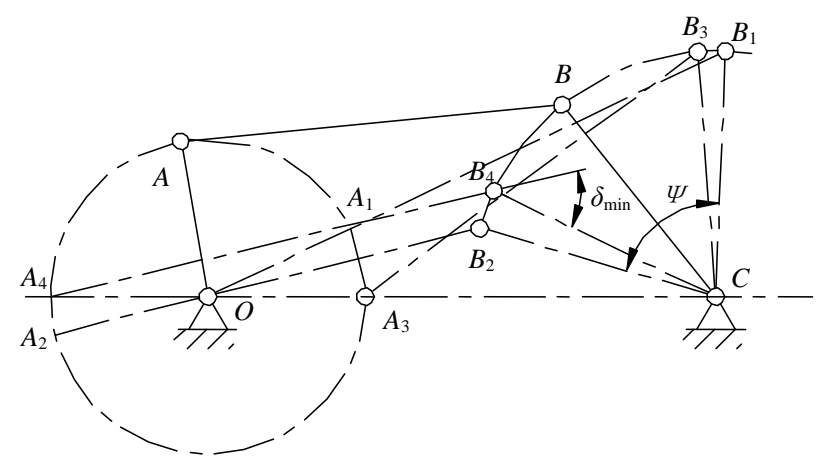

Figure 2. Schematic diagram of II type crank rocker mechanism

\section{SimUlation AND DiscUSSION}

Based on the mechanical dynamics analysis software ADAMS to generate the kinetic model of the crank rocker mechanism, meanwhile, the kinematics characteristics of the mechanism was analyzed ADAMS software can mix design and technology analysis of parts together to construct the overall model of mechanism in the computer, and perform simulation analysis of the product before production, predict the performance, range of motion, collision detection, etc. of mechanical system[7].

According to system model, the software- ADAMS could automatically established Lagrange equation based on the dynamic theory of multi-body system, it can list six Lagrange equation and constraint equation of generalized coordinates with multiplier for each component.

$$
\left.\begin{array}{l}
\&-v_{x}=0 \\
\&-v_{y}=0 \\
\&-v_{z}=0 \\
\&-\omega_{\psi}=0 \\
\&-\omega_{\theta}=0 \\
\&-\omega_{\varphi}=0
\end{array}\right\}
$$

Constraints algebraic equations, external force equation and custom algebraic differential equations are shown in Eq.2.

$$
\left.\begin{array}{l}
\varphi(\& q, t)=0 \\
F(\& u, q, f, t)=0 \\
\operatorname{diff}(\iota \& u, q, f, t)=0
\end{array}\right\}
$$

Where, $\mathrm{q}$ is the generalized coordinate, $\mathrm{u}$ is the differential of each generalized coordinates, $\mathrm{f}$ is the system external forces and constraints.

Make ${ }^{y=[q, u]^{T}}$ as a state vector, the equations of system can be expressed as

$$
G(y, \&, t)=0
$$

\section{A. Parametric model}

During the virtual design, modify and optimize of part structure often lead to re-establish and define of the computing model, this duplication work waste a lot of time. [8] Parametric model organically combines the parametric method and the characteristics of the technology, which greatly improves the efficiency of optimization design. The parametric model is able to calculate all kinds of relationship between feature model of part. When some parts structure of the model is revised, the calculation model only needs to read modify parameters of part structure feature, which can be recalculated to obtain the data more quickly. This article will use the feature technology to establish dynamic simulation model parameterization of the crank rocker mechanism to save the optimization time.

According to the structure of the crank rocker mechanism, the establishment of position of each hinge point $\mathrm{O}, \mathrm{A}, \mathrm{B}, \mathrm{C}$. by the order of point tables. Then, the establishment of 4 design variables DV_1, DV_2, DV_3, DV_4 on behalf of the coordinates of each hinge point. The parameters of each hinge point is shown in table 1 .

TABLE I. PARAMETERS OF EACH POINT

\begin{tabular}{|c|c|c|c|}
\hline Point & Loc_X & Loc_Y & Loc_Z \\
\hline O & 0.0 & 0.0 & 0.0 \\
\hline A & 0.0 & DV_1 & 0.0 \\
\hline B & DV_2 & DV_3 & 0.0 \\
\hline C & DV_4 & 0.0 & 0.0 \\
\hline
\end{tabular}

Click "OK" button, the system automatically creates four hinge points in ADAMS window. Select the geometric modeling tools "link" in the toolbar, set the parameters "New part", establish a crank with the left mouse button to select point $\mathrm{O}$ and point $\mathrm{A}$. Repeat the operation in order to establish connecting rod, rocker and frame.

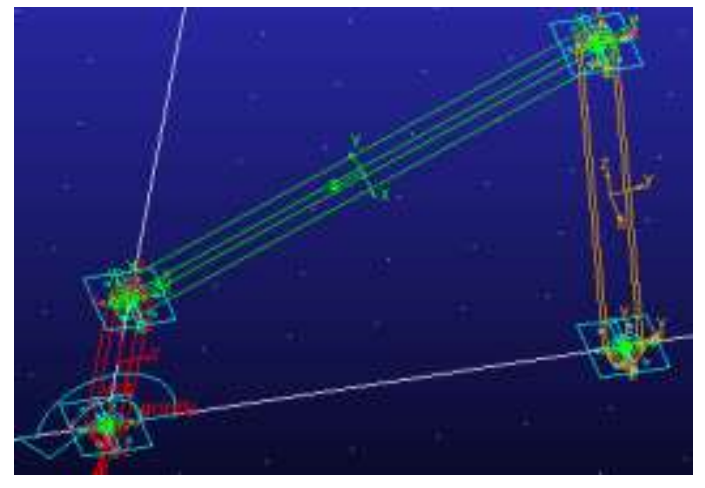

Figure 3. simulation model of I type crank rocker mechanism

Constraint is to determine the relationship of attachment and the relative motion between parts, this is the important point to analysis of kinematics, it is directly affect the accuracy of the analysis and simulation results that the constraints applying is correct or not. Then Gravity 
constraints are applied to the entire mechanism, rotation constraints are added between two members of the crank and the earth, the crank and the connecting rod, the connecting rod and the rocker, the rocker and the earth. The drive is applied on the crank, and the angular velocity is set to $1 \mathrm{rad} / \mathrm{s}$. The simulation model is shown in Fig .3.

\section{B. Optimization of the Transmission Angle}

Transmission angle is an important parameter in the crank rocker mechanical design and denotes the quality of motion transmission in a mechanism. According to the definition of transmission angle, the greater the transmission angle of mechanism, the higher the transmission efficiency. By this reason, the design in the premise of satisfy the known design conditions hope the minimum transmission angle designed of a crank rocker mechanism has a maximum value[9].

A measurement of angle "MEA_ANGLE_1" is arranged between the connecting rod and rocker, then take the maximum of this measurement as the objective function.

Assuming the crank as the active component to do constant velocity rotation, rocker as the follower to swing back and forth, the crank is strictly shortest[10]. At this time for the I type mechanism, constraint functions are expressed as follows

$$
\left\{\begin{array}{l}
g_{1}=L_{1}+L_{4}-L_{2}-L_{3} \leq 0 \\
g_{2}=L_{1}^{2}+L_{4}^{2}-L_{2}^{2}-L_{3}^{2} \leq 0 \\
g_{3}=L_{1}-L_{2} \leq 0 \\
g_{4}=L_{1}-L_{3} \leq 0 \\
g_{4}=L_{1}-L_{4} \leq 0
\end{array}\right.
$$

Create the functions represent each constraint function in ADAMS, respectively. Assuming the swing angle of the crank rocker mechanism of $\Psi=60$, the length of rocker $\mathrm{L}_{3}=250$, the work scope of the crank rocker mechanism model is $80 \mathrm{~mm} \leq L_{1} \leq 120 \mathrm{~mm}, 250 \mathrm{~mm} \leq L_{2} \leq 330 \mathrm{~mm}$ and $250 \mathrm{~mm} \leq L_{4} \leq 350 \mathrm{~mm}$.

Using ADAMS software to optimize the model, step number is 150 . The curve of each iteration value of the objective function is shown in Fig .4, it can be seen from the figure that after 5 iterations, the optimized values of the mechanism is obtained, the value is $42.3175^{\circ}$, which is $4.48 \%$ larger than the original mechanism. In this case the length of each rod are $L_{1}=120, L_{2}=325.72, L_{4}=339.44$. Transmission angle contrast curve before and after optimization is shown in Fig .5.

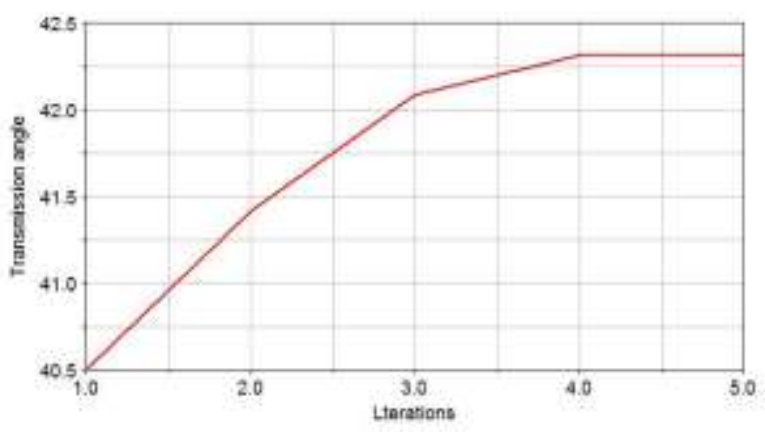

Figure 4. Optimization iteration graph of I type

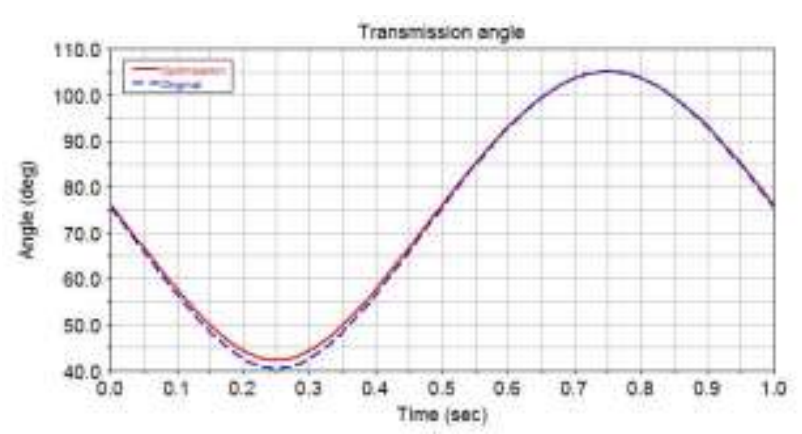

Figure 5. Contrast curve before and after optimization

According to the preceding analysis, for the II type mechanism, a measurement function "180MEA_ANGLE_1" is established, then take the maximum of this measurement as the objective function. Similarly, for the II type mechanism, constraint functions are expressed as follows

$$
\left\{\begin{array}{l}
g_{1}=L_{1}+L_{4}-L_{2}-L_{3} \leq 0 \\
g_{2}=-L_{1}^{2}-L_{4}^{2}+L_{2}^{2}+L_{3}^{2} \leq 0 \\
g_{3}=L_{1}-L_{2} \leq 0 \\
g_{4}=L_{1}-L_{3} \leq 0 \\
g_{4}=L_{1}-L_{4} \leq 0
\end{array}\right.
$$

Assuming the swing angle of the crank rocker mechanism of $\Psi=65$, the length of rocker $L_{3}=290$, the work scope of the crank rocker mechanism model is 120 $\mathrm{mm} \leq L_{1} \leq 180 \mathrm{~mm}, 256 \mathrm{~mm} \leq L_{2} \leq 376 \mathrm{~mm}, 360 \mathrm{~mm} \leq L_{4}$ $\leq 540 \mathrm{~mm}$. The simulation model of II type measurement is shown in Fig .6.

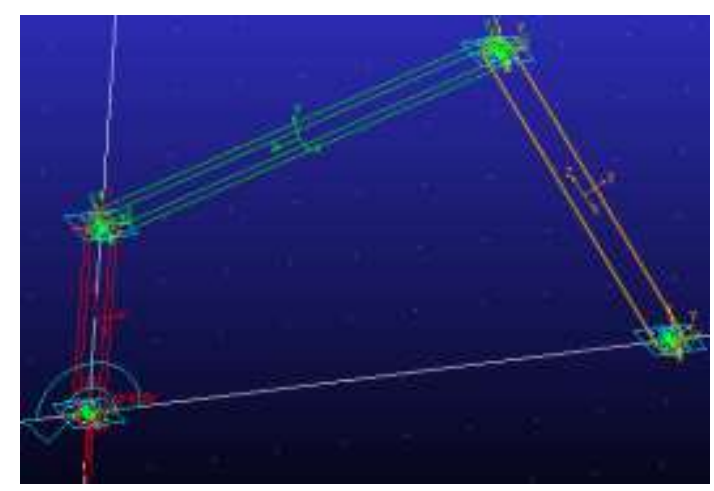

Figure 6. simulation model of II type crank rocker mechanism tep number is 150 . The curve of each iteration value of the objective function is shown in Fig .7, it can be seen from the figure that after 7 iterations, the optimized values of the mechanism is obtained, the value is $50.395^{\circ}$, which is $39.4 \%$ larger than the original mechanism. In this case the length of each rod are $L_{1}=155.82, L_{2}=379.29, L_{4}=451.312$. Transmission angle contrast curve before and after optimization of II type measurement is shown in Fig .8. 


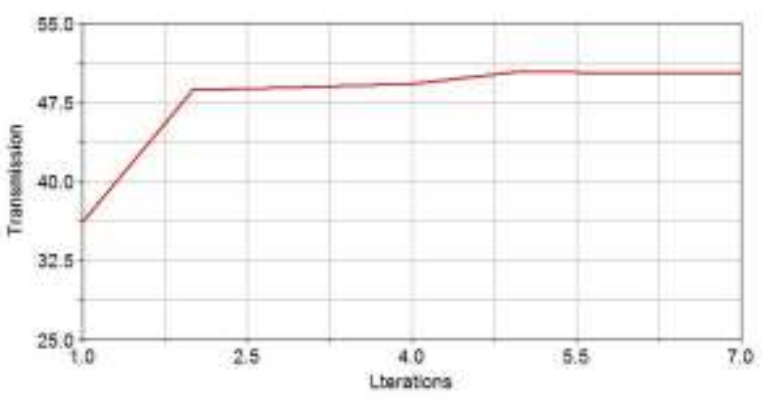

Figure 7. Optimization iteration graph of II type

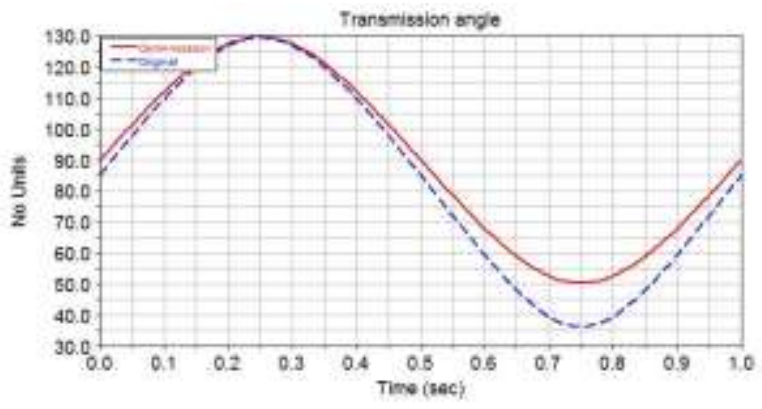

Figure 8. Contrast curve before and after optimization

\section{CONCLUSIONS}

Transmission angle is an important parameter in the crank rocker mechanical design and denotes the quality of motion transmission in a mechanism. In this paper, the structure characteristic of the crank rocker mechanism is analyzed by the graphic principle. The parametric model of I type and II type crank rocker mechanism are established by the dynamics analysis software-ADAMS, respectively. Through optimization,. the minimum transmission angle of
I type mechanism increases by $4.48 \%$, the minimum transmission angle of II type mechanism increases by $39.4 \%$, which can improve the transmission efficiency of the mechanism. This study could provide a reference for the research of movement precision and mechanical design for the mechanism.

\section{REFERENCES}

[1] X. Long, "Design Analysis and Simulation of Crank-rocker Mechanism in Packaging Machinery," PACKAGING ENGINEERING, vol. 35, May. 2014, pp. 76-79.

[2] Z. Fei, H. Zhichao, and L. Jie. "Design approach for crank rocker mechanism with specific rod length," JOURNAL OF MACHINE DESIGN, vol. 31

[3] L. Hongliang, H. Yuemin, and H. Min, "Determination of the Connecting Rod Length of the Crank-rocker Mechanism," Mechanical Engineer, Feb. 2014, pp. 20-22.

[4] L. Zhigang, "Optimized analysis of crank and rocker mechanism based on MATLAB," FOOD \& MACHINERY, vol. 30, Mar. 2014, pp. 114-117.

[5] G.Weidong, Theory of Machines and mechanisms, China Science Press, 2010

[6] C. Ru, "Optimization design of the crank and rocker mechanism based on parameter graphic methed," Journal of Chinese Agricultural Mechanization, vol. 36, Jan. 2015, pp. 50-53.

[7] L.jixia, From entry to the master of the virtual prototype of ADAMS 2012, machine-building industry press, 2013.

[8] S.Youliang, "Optimization design of crank rocker mechanism basede on maximum of minimum transmission angle," JOURNAL OF MACHINE DESIGN, vol. 31, Jul. 2014, pp. 29-33.

[9] T. Xuemei, "The Script Simulation of Shifting Process in Transmission System," International Conference on Mechanic Automation and Control Engineering, 2010.

[10] Y. Jinhu, "Research of the Transmission Angle Function of Offset Crank Rocker Mechanism," Journal of Mechanical, vol. 38, Aug. 2014, pp. 43-46. 